\title{
Standardized low-resolution brain electromagnetic tomography (sLORETA) in the prediction of response to cholinesterase inhibitors in patients with Alzheimer's disease
} Martin Brunovsky*1,2,3, Vladimir Krajca ${ }^{3}$, Frantiska Diblikova ${ }^{1,3}$, Ales Bartos ${ }^{1,2,4}$, Lucie Zavesicka ${ }^{1,2}$ and Milos Matousek ${ }^{1}$

Address: ${ }^{2}$ Prague Psychiatric Center, Prague, Czech Republic, ${ }^{23 r d}$ Medical Faculty, Charles University, Prague, Czech Republic, ${ }^{3}$ Department of Neurology, Faculty Hospital Bulovka, Prague, Czech Republic and ${ }^{4}$ Department of Neurology, Faculty Hospital Kralovske Vinohrady, Prague, Czech Republic

* Corresponding author

from International Society on Brain and Behaviour: 3rd International Congress on Brain and Behaviour

Thessaloniki, Greece. 28 November - 2 December 2007

Published: 17 April 2008

Annals of General Psychiatry 2008, 7(Suppl I):S277 doi:I0.II86/I744-859X-7-SI-S277

This abstract is available from: http://www.annals-general-psychiatry.com/content/7/SI/S277

(c) 2008 Brunovsky et al.; licensee BioMed Central Ltd.

\section{Background}

We tried to identify subgroup of patients with Alzheimer's disease (AD) benefiting from cholinesterase inhibitors (ChEI) treatment using standardized low-resolution brain electromagnetic tomography (sLORETA) [1], which allows to study cortical EEG sources in 6239 cortical grey matter voxels.

\section{Materials and methods}

Resting EEG was recorded in 20 mild to moderate AD patients (mean age $=75.04$ years; 13 women and 7 men; MMSE 15-24) before and after 6 months and 2 years on ChEI (donepezil, rivastigmine, galantamine) treatment. Based on changes of MMSE scores after 2 years follow-up, 11 patients were classified as Non-responders (decrease of MMSE > 2) and 9 patients as Responders (decrease of MMSE <2). The localization of the differences in activity between two groups (at baseline) and within groups (baseline vs. 6 months) was assessed by voxel-by-voxel ttests of the sLORETA images of the log-transformed computed current density power in seven frequency bands.

\section{Results}

At baseline, Non-responders had significantly greater current densities in delta and alpha frequency band, which sLORETA localized in frontal (BA 6,8,9,32; alpha) and parieto-occipital (BA 7,17,39,40; delta) areas. After 6 months of ChEI treatment only Responders showed an increase of beta current densities, mainly in left frontal and temporal cortex.

\section{Conclusions}

Our results suggest that there is a subgroup of AD patients (probably with more pronounced central cholinergic deficiency syndrome) with better response to ChEI treatment, which can be identified by means of new quantitative EEG technique (sLORETA).

\section{Acknowledgements}

The study was supported by a grant from Ministry of Health of Czech Republic No. IA/8600 - 4.

\section{References}

I. Pascual-Marqui RD: Standardized low-resolution brain electromagnetic tomography (sLORETA): technical details. Methods Find Exp Clin Pharmacol 2002, 24D:5-12. 